\title{
Using laser remote heating to simulate extreme thermal heat loads on nuclear fuels
}

\author{
T. Vidal*, L. Gallais, J. Faucheux, H. Capdevila, Y. Pontillon \\ * Aix Marseille University, CNRS, Centrale Marseille, Institute Fresnel, Marseille, France \\ vidal@fresnel.fr
}

\begin{abstract}
Up to now, predicting accurately the Fission Gas Release (FGR) from high burn up $\mathrm{UO}_{2}$ and/or MOX (Mixed Oxide) fuels at off-normal conditions, such as power transient, reactivity-initiated accident (RIA) and loss-of-coolant accident (LOCA), is still a significant and very challenging task. For this purpose, different $R \& D$ programs have been carried out in France, as well as in other countries. This has been done with a specific emphasis on mechanisms which promote the FGR under accidental conditions. These studies can be performed thanks to dedicated integral experiments conducted in-pile (i.e. in Materials Testing Reactor) with the corresponding cost and constraints, or at the laboratory scale with annealing tests which allow to be representative of specific parameters (thermal history for instance). During these annealing tests under wellknown conditions (temperature, atmosphere), both the absolute level and the time dependence of the released gases should be monitored, together with the corresponding fuel microstructural changes, since experimental knowledge of fission gas release alone is not efficient enough. This approach requires more and more accurate on-line measurements. This corresponds to the driving force of the present work.
\end{abstract}

In this contribution, we will present our progress in developing an experimental platform that can submit nuclear fuel and cladding samples to annealing tests involving very high temperatures (up to $2500^{\circ} \mathrm{C}$ ) and very fast temperature ramp (up to thousands of ${ }^{\circ} \mathrm{C} / \mathrm{s}$ ) with controlled thermal gradients and temporal dynamics. This new platform implements innovative instrumentation, such as optical diagnostics to measure fuel fragmentation kinetics and infrared pyrometry for temperature monitoring. This experiment is based on a high-power laser $(1.5 \mathrm{~kW})$ coupled to an experimental chamber with controlled atmosphere (Ar, $\mathrm{N}_{2}$, or vacuum) and specific optical components.

Based on the spatial beam profile and temporal power function of the laser, it is possible which such a system to produce complex spatio-temporal temperature gradients, relevant for addressing different research needs. It provides access to extreme conditions that are very difficult to reach with other means. Particularly, one of main objectives of this work is to investigate conditions of Reactivity Initiated Accident (RIA).

The first experiments performed on inactive materials, nonirradiated uranium dioxide, is presented in order to highlight the capabilities of this technique.

Keywords- Nuclear fuel, Laser Heating, Simulation, Experimentation, High temperature, Instrumentation

\section{INTRODUCTION}

The understanding of the behaviour of nuclear materials in relation to the different thermal loads to which they can be subjected, i.e. from nominal operation through extreme

This work has been partly funded by EdF and Framatome under the CEA PJIPG's program. The authors would like to thank S. Gossé and A. Quaini from CEA Saclay for their help with temperature profile measurements. conditions, is of paramount importance in the nuclear field. By improving this knowledge, it is possible to improve nuclear reactor safety and to precisely define specific on-site emergency plans in the case of an extreme event. Up to now, predicting correctly the fission gas (FG), fission product (FP) releases (i.e. the source term) and the corresponding fuel fragmentation from nuclear fuels at off-normal conditions, such as power transient, reactivity initiated accident (RIA) and loss-of-coolant accident (LOCA), remains a significant and important challenge. In the specific case of the so-called RIA [1], a fast rise in fuel power and temperature occurs. The power excursion may lead to failure of the nuclear fuel rods and release of radioactive material into the primary reactor coolant. In the more severe cases, the fuel rods may be shattered and large amount of the fuel pellet inventory could be dispersed into the primary coolant. To prevent those potential consequences, safety criteria are usually set up to limit the energy injection into the fuel. In such a situation the temperature gradient evolves from nominal situation to an almost flat top radial profile with a maximum localized in an outer ring closed to the pellet periphery [2]. Typical increase above $2000^{\circ} \mathrm{C}$ occurs in few tens of millisecond on the edges of the fuel as described on the simulation reported in [2].

Historically, the worst reactivity-initiated accident took place on April 261986 in reactor 4 of the Chernobyl nuclear power plant in Ukraine. The Chernobyl accident reminded the nuclear community of the destructive potential of RIAs, and it prompted much research into the subject. In order to study the impact of this particular accident, in the early 1990s, experimental programs, in the form of pulse irradiation tests performed in Material Testing Reactor (MTR), were launched in France, Japan and Russia in order to check high burn-up fuel behaviour under RIA. These test programs were primarily intended to check the adequacy of regulatory acceptance criteria for RIA, which were mainly based on experiment on low or un-irradiated fuel. Therefore, up to now, integral experiments (i.e. MTR type) are most often the only solution for this category of R\&D. This means that the potential number of experiments that can be performed is very limited both in terms of their cost and their complexity. To overcome these difficulties, the present reported work aims at exploring a concept to reproduce the so-called RIA (Reactivity Initiated Accident) thermal conditions to study the behaviour of nuclear fuel in such situations. The goal is to provide complementary results to those obtained under integral experiment such as the CIP program (Cabri

T. Vidal and L. Gallais are with the Institut Fresnel, Aix Marseille Univ, CNRS, Centrale Marseille, France (e-mail: laurent.gallais@fresnel.fr).

J. Faucheux, H. Capdevila, Y. Pontillon are with the CEA DEN, SaintPaul-Lez-Durance, France (e-mail: yves.pontillon@cea.fr). 
International Program) which studies the capacity of Pressurized Water Reactor (PWR) fuel based on uranium dioxide $\left(\mathrm{UO}_{2}\right)$ and mixed oxide fuel (MOX) to withstand sudden power transients that may occur during an accident scenario [3].

\section{CONCEPT OF LASER CONTROLLED RIA THERMAL TRANSIENT EXPERIMENT}

\section{A. Laser heating approach}

In order to produce the described RIA thermal transients on nuclear fuel samples at the laboratory scale, we have explored the possibility to use a laser heating approach. Laser remote heating has many very interesting characteristics for such application: (1) it is a contactless technique which can easily drive materials to extreme temperatures with very high spatial precision and temporal control; (2) with focused beam application, the sample being tested essentially acts as its own containment vessel, avoiding any wall contaminants, and virtually without affecting the surroundings because only a small part of the material is being treated; (3) in combination with infrared imaging the measurement is less affected by thermal radiation that should occur from the containment vessel; (4) because of the possible optical access to the sample, laser heating can be combined to several non-contact diagnostics such as infrared imaging to derive meaningful thermo-physical properties avoiding interferences that would normally occur with direct contact measurements of temperature. In the field of nuclear fission research, there are several works dealing with the use of high power laser / material interactions for the investigation of the properties of fuel materials at high temperature, starting from the 80's [4,5]. Particularly, laser heating devices together with analysis diagnostics and temperature monitoring systems have been applied for the measurement of thermal properties (heat capacity, diffusivity, conductivity) [6] and thermodynamic properties (melting point, evaporation point, vapor pressure, equation of state) [7-15]. Combined laser heating with sample levitation and synchrotron x-rays has also allowed studying molten uranium dioxide structure at very high temperature (up to $3270 \mathrm{~K}$ ) [16]. There is clearly a high potential of using such techniques for fuel material research and we report in the following on our first steps in that direction.

\section{B. The proposed concept}

Nuclear fuels being highly absorbing materials to visible/IR wavelengths, the laser flux can be efficiently absorbed by the material. Based on the optical properties of $\mathrm{UO}_{2}$ [17] laser absorption is localized only at the surface of the material and the laser heat source can only be used as an incoming heat flux on the sample surface. Experiments have then to be conceived accordingly. On the thermal point of view, considering a thermal diffusion length of $2 \sqrt{D t}$ with $\mathrm{D}$ the diffusivity of the material and the time of applied laser heating, it occurs that with a diffusivity of $2 \times 10^{-6} \mathrm{~m}^{2} \mathrm{~s}^{-1}[18]$, homogenous heating in the depth of the material at the time scale of few tens of millisecond, corresponding to RIA temporal dynamics, is only obtained if the width of the sample is sub-millimetric. Therefore, in order to conduct relevant laser heating experiments on fuel materials, the samples should be thin disks of less than $1 \mathrm{~mm}$ thickness cut from a fuel pellet if one want to reach homogenous heating in the depth. In such conditions, the proposed scenario to reproduce the RIA spatio-temporal thermal transient is described schematically in Fig. 1: Based on a section of a fuel cladding assembly, the sample is maintained in nominal temperature conditions which can be done through laser heating with flat-top or Gaussian intensity distribution and adapted insulation/cooling. Then annular laser beams, coming from both sides, can be applied to reproduce the temperature gradients encountered in RIA.

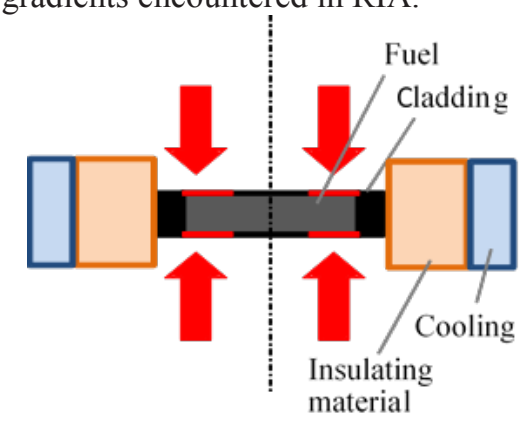

Fig. 1. Concept of laser simulated RIA conditions (The axis shows the axial symetry)

\section{Required laser power}

The dimensioning of such an experiment has been achieved by numerical simulations with COMSOL Multiphysics Software (Finite Elements Method). Depending on the required spatial gradients and temporal dynamics, the laser beam and temporal profile can be adjusted to reach the required conditions. With a typical thickness of the disk sample in the range of $0.5-1 \mathrm{~mm}$, few hundreds Watts of laser power for the transient annular laser beam heating are required to reach temperature close to $2000^{\circ} \mathrm{C}$ in few tens of milliseconds (Example Fig. 2).

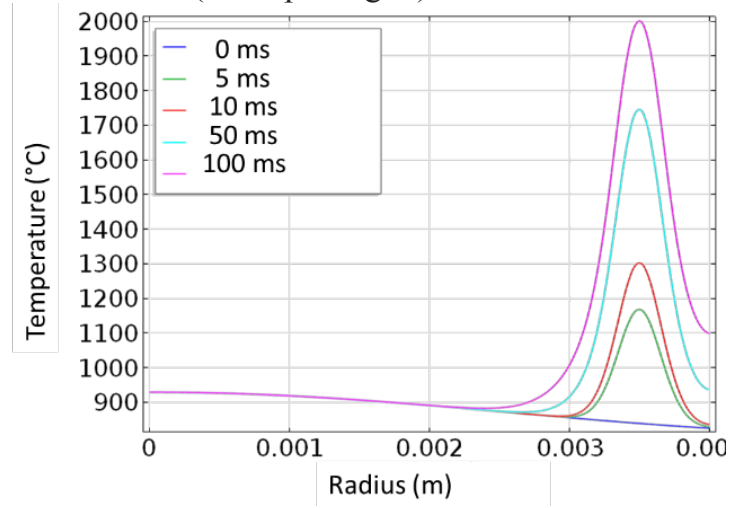

Fig. 2. Simulation of radial temperature distribution at the surface of a $\mathrm{UO}_{2}$ disk submitted to $300 \mathrm{~W}$ annular laser beam heating during $100 \mathrm{~ms}$ (in the configuration shown on Fig. 1). The initial tempeature in this example is $900^{\circ} \mathrm{C}$.

\section{EXPERIMENTAL PLATFORM}

\section{A. Experimental configuration}

Following previous description of the targeted experiment a high power laser delivering hundreds of Watts is required, with the ability to generate millisecond pulses. We have chosen to work with high power Ytterbium fiber lasers (wavelength of $1080 \mathrm{~nm}$ ) that can deliver monomode laser beams (high optical quality for annular beam generation) with $\mathrm{kW}$ output power and typical rising time of few microseconds. 
We have designed a specific sample holder for the experiments, described on Fig. 3. The thin disk sample is placed at the center of an experimental vacuum chamber in a mechanical assembly that has been designed to hold the sample vertically, with insulating materials, zirconia and alumina, that can withstand high temperatures (up to 2000 ${ }^{\circ} \mathrm{C}$ ). Sapphire optical windows are used to maintain the sample and potentially retain the fragments in place in case of loss of integrity, while simultaneously allowing optical access from both sides for laser beams and optical instrumentation.

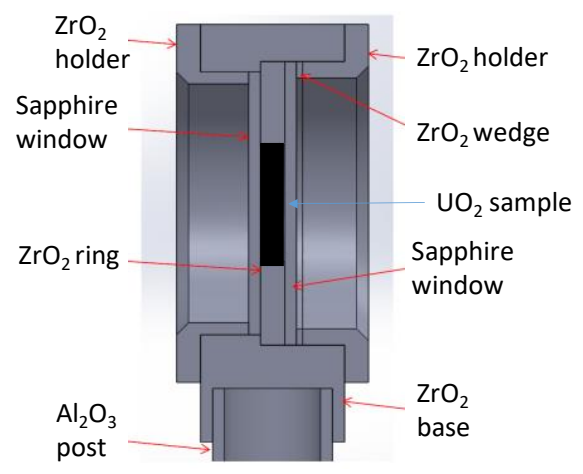

Fig. 3. Schematic of the sample holder assembly at the center of the vacuum chamber. $\mathrm{A} \mathrm{UO}_{2}$ disk, $8 \mathrm{~mm}$ diameter and $1 \mathrm{~mm}$ thick is visible in the sample holder.

\section{B. Laser beams}

On each side of the sample two different laser beams are used for (1) heating the sample at a nominal temperature corresponding to initial conditions before the transient and (2) generate a transient heat load with annular distribution on the edges of the sample. In order to generate the annular beam for controlled energy deposition on the edges of the sample, a classical lens and positive axicon combination has been used [19]. A $8 \mathrm{~mm}$ beam diameter with a $150 \mu \mathrm{m}$ width is obtained with a $13 \mathrm{~mm}$ incoming Gaussian beam (diameter at $1 / \mathrm{e}^{2}$ ), a $500 \mathrm{~mm}$ focal length and a 1 degree axicon. The image of the obtained ring in such conditions is shown on Fig 4 as measured with a laser beam profiler placed in the sample plane. By defocusing the lens it is possible to adjust the width of the annular beam and accordingly modify the temperature gradients.

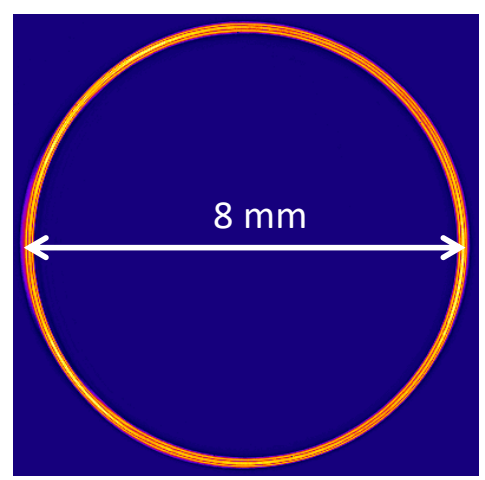

Fig. 4. Image of the beam profile at $150 \mathrm{~W}$ in the sample plane obtained with a high power laser beam profiler.

\section{Instrumentation}

The optical instrumentation around the sample is a key point to monitor and control the temperature, therefore optical diagnostics such as thermal imaging with high speed cameras and optical pyrometry have be implemented through different viewports of the experimental chamber. A picture of the experimental configuration is given on Fig. 5.

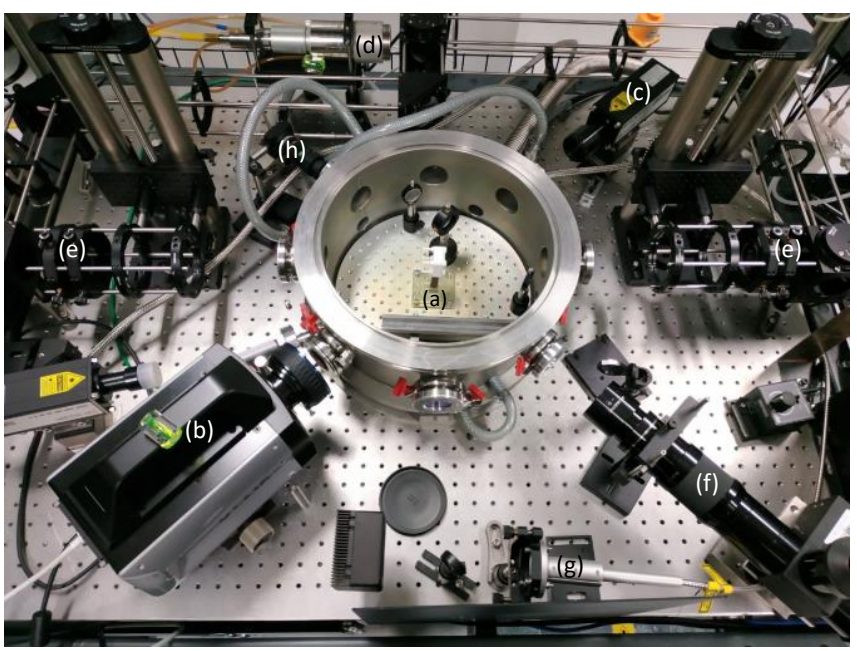

Fig. 5. Picture of the experimental configuration. (a) Sample holder in the center of the opened experimental chamber ; (b) High speed MWIR thermal camera ; (c) Pyrometer ; (d) High power Laser collimator for transient heating; (e) Optical systems for annular beam generation ; (f) long distance microscope ; (g) Medium power laser collimator for continuous heating ; (h) Obervation system for beam alignement.

The instrumentation is based on:

a) Optical access to the sample with transparent parts of the sample holder (sapphire windows) and specific viewports in the experimental chamber (Anti-reflection coated silica, BaF2 or ZnSe windows).

b) A High speed thermal camera which operates in the $3-5 \mu \mathrm{m}$ spectral range, corresponding to the transparency range of the Sapphire windows. This system allows measurements up to $3000^{\circ} \mathrm{C}$ at $1 \mathrm{kHz}$.

c) $\quad A$ pyrometer with fast response time $(<1 \mathrm{~ms})$ operating in the $2-2.6 \mu \mathrm{m}$ spectral range. This system is used to monitor the surface temperature at the center of the sample, which is used in a PID regulation loop to control the laser power and ensure stable temperature.

d) Laser control with a dedicated software that allows generation of arbitrary temporal functions for the transient pulse heating.

e) Beam shaping systems to produce the annular beams. The beam profiles are measured at the sample location before the experiments. Knowledge of the beam profile is required for numerical simulations of the experiments.

f) A long distance microscope coupled with a visible range high speed camera ( $>1000$ frames per second) is used to observe the sample surface in real time and provide additional information to thermal measurements (for instance micro structure evolution and fragmentation). This system has been specifically developed for $\mathrm{UO}_{2}$ observation and is described in [20].

g) PID regulation of a second medium power laser, based on the temperature monitoring of the sample with the pyrometer, in order to ensure stable temperature conditions.

An optical system (CMOS camera + objective) is used to observed the positioning of the laser beam on the sample with respect to the sample. This alignment is indeed critical for the experiments 


\section{RESUlts}

As a preliminary study, we have firstly worked on graphite samples to validate the experimental choices and compare experimental results to numerical ones. This part is not reported in this paper, we refer to [21]. In the following we describe the performances of the system to generate control thermal transients on $\mathrm{UO}_{2}$ samples. The samples used in this work were synthesized in the $\mathrm{UO}_{2}$ laboratory at the CEA Cadarache Center using standard general process: they were synthesized with depleted $\mathrm{UO}_{2}$ from batch produced through wet process, followed by conventional pre-compaction $(50$ $\mathrm{MPa})$, pressing (450 MPa) and sintering at $1650{ }^{\circ} \mathrm{C}$ for $2 \mathrm{~h}$ under flowing $\mathrm{H}_{2}$. The reported experiments have been done in vacuum environment with a base pressure of $2 \times 10^{-2}$ mbar.

\section{A. Initial conditions}

The RIA experiments require to reach the initial temperature conditions before the transient heating, with an accurate monitoring and control of the sample temperature. This is achieved with the heating laser and PID feedback loop described before. The laser beam used for this purpose has a Gaussian spatial profile with $8 \mathrm{~mm}$ diameter (at $1 / \mathrm{e}^{2}$ ) at the sample location in order to cover the whole sample surface and ensure homogeneous heating. The sample is heated only from one side since due the low thickness $(<1 \mathrm{~mm})$ and long irradiation time it is sufficient to obtain a homogeneous temperature in the sample. A stable temperature with variations below $1 \%$ was measured (see example Fig. 6), with heating ramps up to $100^{\circ} \mathrm{C} / \mathrm{s}$ to reach the temperature plateau.

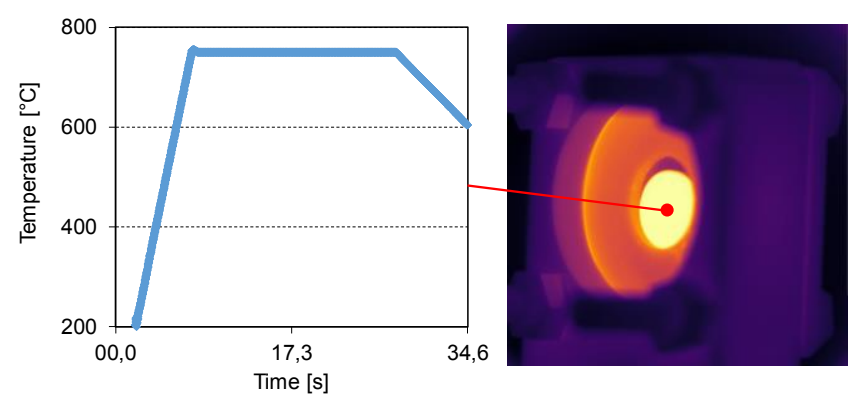

Fig. 6. Example of a regulated laser heating on a $\mathrm{UO}_{2}$ sample. Left: measured temperature with the pyrometer at the sample surface in case of $100^{\circ} \mathrm{C} / \mathrm{s}$ heating ramp followed by $20 \mathrm{~s}$ plateau at $750^{\circ} \mathrm{C}$. Right: Observation of the sample with the thermal camera during the temperature plateau.

\section{B. Transient heating}

Once the initial temperature is reached, the two laser beams with annular spatial profiles can be launched. In the reported experiments the two beams are coming from the same laser source that is divided in two beam paths with a $50 \%$ beam splitter. This ensures synchronization of the 2 beams and power balance. The temperature distribution however was only measured on one side with the thermal camera. We report on Fig. 7 on typical experimental results for illustration of the concept. This experiment has been done with a $20 \mathrm{~ms}$ pulse transient and $600 \mathrm{~W}$ incident power $(300 \mathrm{~W}$ on each face).

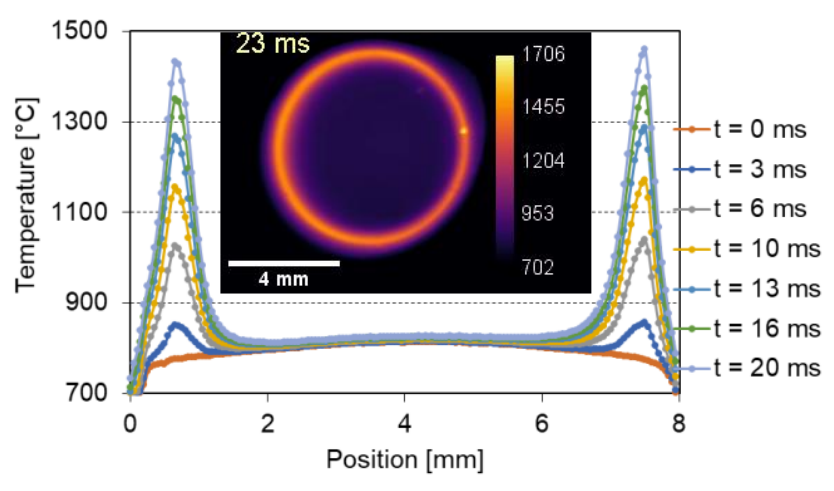

Fig. 7. Measured temperature at the surface of a $\mathrm{UO}_{2}$ sample submitted to $300 \mathrm{~W}$ annular beam irradiation during $20 \mathrm{~ms}$ on both sides. Image: thermal camera snapshot at the end of the laser pulse ; Data plot: extraction of the temperature along vertical profile of the thermal images at different times.

The temperature distribution during the transient has the expected radial symmetry, as illustrated by the good correspondence of the extracted temperature profiles, with peak temperatures corresponding to the required ones to reach RIA conditions. It illustrates the fact that in the proposed experimental conditions, the temperature gradients can be well controlled by the proposed laser technique.

In the different set of experiments that were conducted, fracturation of the samples was however observed in some cases, as shown on the Scanning Electron Microscope images reported in Fig. 8. Fragmentation of edges of the sample was also observed in some extreme cases (transient temperature $>$ $2200{ }^{\circ} \mathrm{C}$ ). The experiments have then also some interest to study thermo-mechanical behaviour of the materials. Thermomechanical simulations conducted with the ALCYONE code indicate that under such laser exposure conditions, sample rupture should occur in agreement with observations.

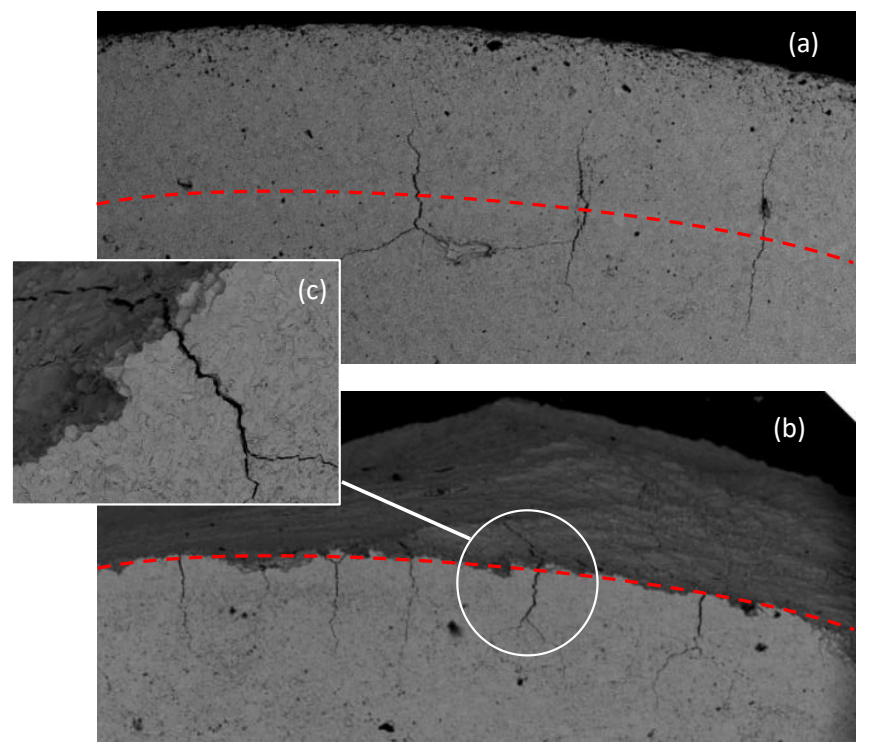

Fig. 8. Scanning Electron Microscope images of samples after the laser experiments. The dotted red line indicates the position of the peak laser intensity. (a) Sample exposed to annular beam of $300 \mathrm{~W}$ during $10 \mathrm{~ms}$; (b) Sample exposed to annular beam of $300 \mathrm{~W}$ during $100 \mathrm{~ms}$; (c) Details of the observed fracture.

\section{CONCLUSION AND PERSPECTIVES}

A concept based on the use of high power lasers has been proposed for the generation of temperature transients on 
nuclear fuels corresponding to Reactivity Initiated Accident conditions. A pilot platform has been developed for the validation of this concept, then as the first phase of hot-cell integration. This pilot platform will be used for the proposal of devices and experimental programs that can be integrated into the hot laboratories at the CEA Cadarache to perform experiments that can meet emerging research needs. Its successful completion will permit, with some further developments, a full real time analysis of the irradiated nuclear fuel behaviour in case of a major mishap, including the release of radioactive gases and particles at high temperature. This will provide crucial experimental data for physical model validation implemented in fuel performance codes.

\section{(1) Acknowledgment}

This work has been partly funded by EdF and Framatome under the CEA PJIPG's program.

The authors would like to thank S. Gossé and A. Quaini from CEA Saclay for their help with temperature profile measurements.

\section{(2) References}

[1] F. Schmitz and J. Papin, "High burnup effects on fuel behavior under accident conditions: the tests CABRI REP-Na," Journal of Nuclear Materials, vol. 270, pp. 55, 1999.

[2] J I. Guenot-Delahaie, J. Sercombe, T. Helfer, P. Goldbronn, E. Federici, T. Le Jolu, A. Parrot, C. Delafoy and C. Bernaudat, "Simulation of reactivity-initiated accident transients on $\mathrm{UO}_{2}-\mathrm{M} 5$ fuelrods with ALCYONE V1.4 fuel performance code," Nuclear Engineering and Technology, vol. 50, pp 268, 2018.

[3] J. Papin, B. Cazalis, J.M. Frizonnet, J. Desquines, F. Lemoine, V. Georgenthum, F. Lamare and M. Petit, "Summary and interpretation of the CABRI REP-Na program," Nuclear Technology, vol. 157, pp 230, 2007.

[4] R.W. Ohse, J.F. Babelot, G.D. Brumme, and P.R. Kinsman, 'Extension of vapor-pressure measurements of nuclear oxide fuels on $\mathrm{UO}_{2}$ and $(\mathrm{U}, \mathrm{Pu}) \mathrm{O}_{2}$ for fast-reactor safety analysis by laser techniques up to $5000 \mathrm{~K}$, Revue internationale des hautes temperatures et des réfractaires, vol. 15, pp 319, 1978.

[5] S.R. Yagnik, D.R. Olander, 'Surface temperature transients from pulsed laser heating of $\mathrm{UO}_{2}$ ', Journal of Nuclear Materials, vol. 154, pp 253, 1988.

[6] C. Ronchi, M. Sheindlin, M. Musella, G. J. Hyland, 'Thermal onductivity of uranium dioxide up to $2900 \mathrm{~K}$ from simultaneous measurement of the heat capacity and thermal diffusivity', Journal of Applied Physics, vol. 85, pp 776, 1999.

[7] C. Ronchi, M. Sheindlin, "Laser-Pulse Melting of Nuclear Refractory Ceramics," Int. J. of Thermophysics, vol; 23, pp 293, 2002.

[8] R. Pflieger, J.-Y. Colle, I. Iosilevskiy, M. Sheindlin, "Urania vapor composition at very high temperatures," Journal of Applied Physics vol. 109, pp 033501, 2011

[9] F. De Bruycker, K. Boboridis, P. Pöml, R. Eloirdi, R.J.M. Konings, D. Manara, "The melting behaviour of plutonium dioxide: A laser-heating study," Journal of Nuclear Materials, vol. 416, pp 166, 2011.

[10] D. Manara, C. Ronchi, M. Sheindlin, "Pressure Dependence of $\mathrm{UO}_{2}$ Melting Measured by Double-Pulse Laser Heating," International Journal of Thermophysics, vol. 23, pp 47, 2002.

[11] M. Joseph, N. Sivakumar, P. Manoravi, "Studies on equation of state of high temperature nuclear materials," Annals of Nuclear Energy, vol. 31, pp 1163, 2004.

[12] P. D. W. Bottomley, Th. Wiss, A. Janssen, B. Cremer, H. Thiele, D. Manara, M. Scheindlin, M. Murray-Farthing, P. Lajarge, M. Menna, D. Bouexière, V. V. Rondinella, "Characterisation of high temperature refractory ceramics for nuclear applications," Materials Science and Engineering, vol. 32, pp 012003, 2012.

[13] D. Manara, R. Böhler, K. Boboridis, L. Capriotti, A. Quaini, L. Luzzi, F. De Bruycker, C. Guéneaud, N. Dupine, R. Konings, "The melting behaviour of oxide nuclear fuels: effects of the oxygen potential studied by laser heating," Procedia Chemistry, vol.7, pp 505, 2012.

[14] F. Cappia, R. Jovani-Abril, J. Spino, L. Luzzi, A. Janßen, D. Manara, "Laser melting of nano-crystalline uranium dioxide," Progress in Nuclear Energy, vol. 72, pp 11-16, 2013.

[15] D. Prieur, F. Lebreton, M. Caisso, P.M. Martin, A.C. Scheinost, T. Delahaye, D. Manara, "Melting behaviour of americium-doped uranium dioxide," J. Chem. Thermodynamics, vol. 97, pp 244, 2016.

[16] L. B. Skinne, C. J. Benmore, J. K. R. Weber, M. A. Williamson, A. Tamalonis, A. Hebden, T. Wiencek, O. L. G. Alderman, M. Guthrie, L. Leibowitz, J. B. Parise, "Molten uranium dioxide structure and dynamics," Science, vol. 345, pp 984, 2014.

[17] M.Bober, J.Singer, K.Wagner, "Determination of the optical constants of molten nuclear fuels," Journal of Nuclear Materials, vol. 124, pp 120, 1984.

[18] L. Vlahovic, D. Staicu, A. Küst, R.J.M. Konings, "Thermal diffusivity of UO2up to the melting point," Journal of Nuclear materials, vol. 499, pp 504, 2018.

[19] M. Rioux, R. Tremblay,P. A. Belanger, "Linear, annular, and radial focusing with axicons and applications to laser machining," Applied Optics, vol. 17, pp 1532, 1978.

[20] T. Vidal, L. Gallais, R. Burla, F. Martin, H. Capdevila, S. Clément, Y. Pontillon, "Optical system for real-time monitoring of nuclear fuel pellets at high temperature," Nuclear Engineering and Design, in press.

[21] T. Vidal, L. Gallais, J. Faucheux, H. Capdevila, J. Sercombe, Y. Pontillon, "Simulation of Reactivity Initiated Accident thermal transients on nuclear fueld with laser remote heating," submitted. 